\title{
On the origin of X-ray flashes
}

\author{
S. Dado ${ }^{1}$, A. Dar ${ }^{1,2}$, and A. De Rújula ${ }^{2}$ \\ ${ }^{1}$ Physics Department and Space Research Institute, Technion, Haifa 32000, Israel \\ e-mail: arnon@physics.technion.ac.il \\ 2 Theory Division, CERN, 1211 Geneva 23, Switzerland
}

Received 7 October 2003 / Accepted 12 February 2004

\begin{abstract}
We use the cannonball (CB) model of gamma ray bursts (GRBs) and their afterglows (AGs) to analyze the observational data on X-ray flashes (XRFs) and their AGs. We show that the observations support the CB-model interpretation that XRFs, like GRBs, are produced by the explosions of core-collapse supernovae (SNe) akin to SN1998bw, by jets of highlyrelativistic CBs. The XRFs and GRBs are intrinsically identical objects, but the XRFs are viewed from angles (relative to the jet direction) which are typically a few times larger than the typical viewing angles of "classical", long-duration GRBs. There should be XRFs, not observed so far, with durations similar to those of short GRBs.
\end{abstract}

Key words. X-rays: bursts $-\gamma$-rays: bursts - stars: supernovae: general

\section{Introduction and summary}

By definition, X-ray flashes (XRFs) are gamma ray bursts (GRBs) whose "peak photon energy", $E_{\mathrm{p}}$, is below $40 \mathrm{keV}$ (roughly speaking, $E_{\mathrm{p}}$ is the maximum of the distribution $\left.v F_{v}=E^{2} \mathrm{~d} N_{\gamma} / \mathrm{d} E\right)$. They are rich in X-rays but relatively poor in $\gamma$-rays, as implied by their name. They were discovered with the Wide-Field Camera of the Beppo-SAX satellite, but they were not seen above $40 \mathrm{keV}$ with its GRB Monitor (Heise et al. 2001). They were detected by BeppoSAX at a rate of 4 per year, indicating a population not very much smaller than that of GRBs ${ }^{1}$. Re-examining the BATSE data, Kippen et al. (2002) have found some 10 XRFs. A few more have been detected by HETE II. To date, about 30 XRFs have been reported. These bursts are distinguished from Galactic transient sources by their isotropic spatial distribution, analogous to that of GRBs. They are softer and dimmer than GRBs, but otherwise their properties are similar: they have similar durations and light curves, their spectrum is also well described by the "Band spectrum" (Band et al. 1993) and their spectral evolution is similar to that of GRBs.

To date, four XRFs (011030: Gandolfi et al. 2001; 020427: in 't Zand et al. 2002; 020903: Ricker et al. 2002; 030723: Prigozhin et al. 2003) have been followed up sufficiently rapidly to allow for the detection of their afterglows (AGs), i.e. continued lower-energy emissions, and for their precise (sub-arcsecond) localization. The AGs of XRFs were first discovered by Taylor et al. (2001) in the radio band, by Harrison et al. (2001) in the X-ray band, and by Soderberg et al. (2002) in the optical band. A host galaxy of an XRF was discovered first

${ }^{1}$ GRBs were detected by BATSE at a rate of $\sim 1$ per day, but with much higher sky-coverage than BeppoSAX has. by Fruchter et al. (2002). The early-time AGs of XRFs have spectral and temporal properties similar to those of the AGs of the "classical" long-duration GRBs, but they are dimmer. In three cases the precise localization afforded by the AG observations led to an identification of the putative host galaxies of the XRFs progenitors (Soderberg et al. 2002; Bloom et al. 2003a). The few available redshift and photometric informations on these host loci indicate that XRFs are cosmological in origin, and that their progenitors, like those of long-duration GRBs, permeate star-formation regions of their host galaxies. Not all measured XRF redshifts are high enough to explain their relatively low peak flux and low peak energy simply on grounds of distance and cosmological redshift.

In the cannonball (CB) model (Dar \& De Rújula 2000, 2003; Dado et al. 2002a, 2003a, and references therein) longduration GRBs and their AGs are produced in ordinary corecollapse supernova (SN) explosions by jets of $\mathrm{CBs}$, made of ordinary atomic matter. Each CB gives rise to a single $\gamma$-ray pulse of a GRB's light curve. The CBs are initially travelling with high Lorentz factors, $\gamma$, which peak very narrowly around an initial $\gamma \sim 10^{3}$ (Dado et al. 2002a, 2003a). The light CBs emit is collimated in a cone of opening angle $\sim 1 / \gamma \sim 1 \mathrm{mrad}$. The "jet opening angle" $\theta_{v}$ (subtended by a CBs' transverse radius as viewed from their point of emission) is smaller than $1 / \gamma$ and its effects can be neglected: in this respect, it is as if CBs were point-like. The differences between the pulses of different GRBs are dominated by the different values of $\theta$, the observer's viewing angle relative to the CB's direction of motion (Dar \& De Rújula 2000, 2003).

The properties of XRFs are similar to those of GRB 980425, which, in the CB model, was interpreted (Dar \& De Rújula 2000, 2003; Dado et al. 2002a, 2003a) as an 
entirely normal GRB produced by the explosion of SN1998bw (Galama et al. 1998). Its jet of CBs was ejected at an angle $\theta \sim 3.9 / \gamma \sim 8 \mathrm{mrad}$, a large value which, combined with the progenitor's unusually small redshift $(z=0.0085)$ conspired to produce a rather typical GRB fluence (Dar \& De Rújula 2000, 2003; Dado et al. 2002a, 2003a). GRB 980425 is by definition a GRB and not an XRF, as the central value of its peak energy, $E_{\mathrm{p}}=54.6 \pm 20.9 \mathrm{keV}$ (Yamazaki et al. 2003a), is just above the "official" borderline $40 \mathrm{keV}$. In the CB model, SN1998bw, associated with GRB 980425, is an ordinary core-collapse SN: its "peculiar" X-ray and radio emissions were not emitted by the SN, but were part of the GRB's AG (Dado et al. 2002a, 2003a). The high velocity of its ejecta is attributed to the SN being viewed almost "on axis".

The CB-model interpretation of XRFs (Dar \& De Rújula 2003 ) is entirely straightforward: XRFs are GRBs viewed further off axis ${ }^{2}$. Thus, all of the CB-model results (e.g., Dado et al. 2002a, 2003a) are applicable to XRFs.

In this paper we show that the CB model explains well all the observed properties of XRFs and their AGs. In particular, their large viewing angles result in much smaller Doppler factors, $\delta$, than those of GRBs. For the Lorentz factors and viewing angles relevant to our discussion, $\gamma^{2} \gg 1$ and $\theta^{2} \ll 1$, and

$\delta=\frac{1}{\gamma(1-\beta \cos \theta)} \approx \frac{2 \gamma}{1+\gamma^{2} \theta^{2}}$,

to an excellent approximation.

Relative to GRBs, XRFs have pulses that are dimmer in fluence by a factor $\delta^{3}$ (or in photon number-count by a factor $\delta^{2}$ ). The XRF pulses are wider and their peak energies are smaller than those of GRBs, both by a factor $\delta$. Their AGs are dimmer and less rapidly-evolving at early times, in a manner that we shall describe. On the other hand, both for XRFs and GRBs, the total duration of multi-pulse events is governed by the properties of the engine generating the CBs: the observed duration and the local duration at the progenitor's position are related by a cosmological time-stretching factor, $1+z$, which does not involve $\delta$. The total duration of XRFs is therefore akin to that of GRBs, while their structure is - since they consist of similar numbers of wider pulses - smoother than that of GRBs. Finally, in the AGs of relatively nearby XRFs it should be possible to observe the contribution of a SN akin to SN1998bw, displaced to the XRF's position and peaking about one month after the SN exploded and the XRF was emitted (Dar \& De Rújula 2003). Such a shining-gun signature may already have been observed in the AG of XRF 030723 (Fynbo et al. 2003), see Fig. 6.

\footnotetext{
2 Subsequent to critiques (Dar 1998, 1999, 2003; Dar \& Plaga 1999; Dado et al. 2002a; De Rújula 2002, 2003) of the on-axis viewingangle anzatz, $\theta=0$, of the "collimated-fireball" or conical-jet models (e.g., Rhoads 1997, 1999; Sari et al. 1999; Piran 2000; Panaitescu \& Kumar 2000; Frail et al. 2001; Berger et al. 2003; Bloom et al. 2003b), this assumption has recently been replaced by a $\theta \neq 0$ of uniform or structured conical jets (e.g., Rossi et al. 2002; Granot et al. 2002; Zhang \& Mészáros 2002; Panaitescu \& Kumar 2003; Jin \& Wei 2003; Salmonson 2003). The jets' opening angles are $\theta_{v}>1 / \gamma$, but getting close to inverting the inequality (Waxman 2003; Lazzatti et al. 2003) to spouse the CB-model's geometry.
}

In the CB model, X-ray rich GRBs fall naturally in an intermediate domain between GRBs and XRFs: they do not require a separate discussion.

The interpretation of XRFs as GRBs viewed off axis has also been advocated by other authors (e.g., Yamazaki et al. 2002, 2003b; Jin \& Wei 2003). One main difference between our CB-model and theirs is the jet geometry: effectively pointlike CBs, versus conical shells. Another one is the underlying GRB and XRF production mechanism: inverse Compton scattering by the CBs' electrons of the "ambient light" that permeates the wind-fed surroundings of the parent star, versus synchrotron radiation emitted in collisions of conical shells of a delicately baryon-loaded $\mathrm{e}^{+} \mathrm{e}^{-}$plasma, produced by "collapsars" (Woosley \& MacFadyen 1999) or "hypernovae" (Iwamoto et al. 1998). Finally, the AGs are in both models generated by synchrotron radiation, but in the CB model the process does not involve shocks (Dar \& De Rújula 2003) and results in a description of AG light-curves and wide-band spectra simpler and more successful than that of the fireball or firecone models (Dado et al. 2002a, 2003a, and, for a direct comparison, 2003d).

\section{The engine}

In the $\mathrm{CB}$ model, long-duration GRBs are produced in the explosions of ordinary core-collapse SNe. Following the collapse of the stellar core into a neutron star or a black hole, and given the characteristically large specific angular momentum of stars, an accretion disk or torus is hypothesized to be produced around the newly formed compact object, either by stellar material originally close to the surface of the imploding core and left behind by the explosion-generating outgoing shock, or by more distant stellar matter falling back after its passage (De Rújula 1987). A CB is emitted, as observed in microquasars, when part of the accretion disk falls abruptly onto the compact object (e.g., Mirabel \& Rodrigez 1999; Rodriguez \& Mirabel 1999, and references therein). The high-energy photons of a single pulse in a GRB or an XRF are produced as a CB coasts through the "ambient light" permeating the surroundings of the parent $\mathrm{SN}$. The electrons enclosed in the CB Compton up-scatter photons to energies that, close to the CBs direction of motion, correspond to the $\gamma$-rays of a GRB and less close to it, to the X-rays of an XRF. Each pulse of a GRB or an XRF corresponds to one $\mathrm{CB}$. The timing sequence of emission of the successive individual pulses (or CBs) reflects the chaotic accretion process and its properties are not predictable, but those of the single pulses are (Dar \& De Rújula 2003, and references therein).

The observational evidence prior to GRB 030329 for the claim that core-collapse $\mathrm{SNe}$ are the engines generating GRBs, (e.g., Dar \& Plaga 1999; Dar \& De Rújula 2000), is discussed in Dado et al. (2002a, 2003c). The spectroscopic discovery of SN2003dh in the AG of GRB 030329 (Garnavich et al. 2003; Stanek et al. 2003; Hjorth et al. 2003) with a luminosity and spectrum remarkably similar to those of SN1998bw, as predicted by Dado et al. (2003c), has provided the most convincing evidence for the GRB-SN association after GRB980425/SN1998bw. 


\section{Properties of the X-rays of XRFs}

Since, in the CB model, XRFs are GRBs viewed from a larger angle $\theta$, all we have to do to study or predict the properties of the former is to paraphrase, for larger $\theta$, the properties of the latter, for which the $\mathrm{CB}$ model offers a general, simple and predictive description (Dar \& De Rújula 2003).

If core collapse $\mathrm{SNe}$ and their environments were all identical, and if their ejected CBs were also universal in number, mass, Lorentz factor and velocity of expansion, all GRBs would be standard candles and the observed differences between them would only be due to the observer's position, determined by $z$ and the angle of observation, $\theta$. The distribution of Lorentz factors can be inferred both from the study of GRB AGs (Dado et al. 2002a, 2003a), and from that of the GRBs themselves (Dar \& De Rújula 2003). This distribution is very narrowly peaked around $\gamma \simeq 10^{3}$, so that the dependence of the various observables on the wider distribution of $\theta$ values directly translates into their dependence on $\delta(\theta, \gamma)$ at an approximately constant $\theta$. This dependence is strong in various observables, such that it might overwhelm much of the caseby-case variability induced by the distributions of the other parameters.

\subsection{The viewing angle of XRFs}

The mean peak energy of ordinary GRBs is $\left\langle E_{\mathrm{p}}(G R B)\right\rangle \approx$ $250 \mathrm{keV}$ (e.g., Preece et al. 2000; Amati et al. 2002). It is convenient to introduce the quantity:

$\sigma \equiv \frac{\gamma \delta}{10^{6}} \frac{2}{1+z}$

to which the peak energy of GRBs and XRFs is proportional, since $\gamma \delta /(1+z)$ is the factor by which, on average, electrons with a Lorentz factor $\gamma$ boost the energy of a "target photon" of an isotropic distribution into a final photon observed at an angle $\theta$ (Dar \& De Rújula 2003). The mean $\sigma(G R B)$ of GRBs of known $z$ is $\langle\sigma(G R B)\rangle \approx 1$.

The ratio between the peak energy $E_{\mathrm{p}}(X R F)$ of an XRF and the mean peak energy of GRBs is:

$E_{\mathrm{p}}(X R F)=\frac{\sigma(X R F)}{\langle\sigma(G R B)\rangle}\left\langle E_{\mathrm{p}}(G R B)\right\rangle$.

The defining condition of an XRF, $E_{\mathrm{p}}(X R F)<40 \mathrm{keV}$, implies that $\sigma(X R F)$ must be smaller by a factor $\sim 6.25$ than $\langle\sigma(G R B)\rangle \approx 1$. The mean redshift of GRBs, as inferred from the current sample of 32 GRBs with known redshift, is $\langle z\rangle \approx 1$. By use of Eq. (1), and adopting the characteristic value $\gamma \sim$ $10^{3}$, we learn that the typical viewing angles of XRFs satisfy $\theta_{\mathrm{XRF}} \geq 3.4 \mathrm{mrad}$. For GRBs the result is $\langle\theta\rangle_{\mathrm{GRB}} \sim 1 \mathrm{mrad}$ (Dar $\&$ De Rújula 2003).

The dependence of many other GRB observables on the viewing angle or the Doppler factor (Dar \& De Rújula 2000, 2003; Plaga 2001) has direct testable consequences.

\subsection{Polarization}

Inverse Compton scattering of the ambient light by the CBs' electrons linearly polarizes it. The degree of polarization
$\Pi(\theta, \gamma)$ is a function of only the product $\theta \gamma$ and has the universal shape (Shaviv \& Dar 1995; Dar \& De Rújula 2003):

$\Pi(\theta, \gamma) \approx \frac{2 \theta^{2} \gamma^{2}}{1+\theta^{4} \gamma^{4}}$

For XRFs $\theta \gamma \geq 3.4$ and Eq. (4) yields a polarization level, $\Pi \leq 17 \%$. Thus, the CB model predicts a much smaller linear polarization in XRFs than in GRBs, which reach $\Pi \approx 100 \%$ for $\theta \approx 1 / \gamma$.

\subsection{Duration of single pulses of XRFs}

The times, $t^{\prime}$ in the CB's rest frame, $t_{\mathrm{SN}}$ in the SN rest frame, and $t$ as measured by a distant observer, are related via $\mathrm{d} t=$ $(1+z) \mathrm{d} t^{\prime} / \delta=(1+z) \mathrm{d} t_{\mathrm{SN}} / \gamma \delta$. Hence, any time measure $\Delta t$ in a single pulse in a XRF, such as its full width at half maximum, its rise time, its fall time, or its "lag time" (the difference between the pulse peak times in two different energy intervals), is proportional to $(1+z) / \delta$. In a "standard candle" approximation, it can be written as:

$\Delta t(X R F)=\frac{\left\langle\Delta t E_{\mathrm{p}}\right\rangle_{\mathrm{GRB}}}{E_{\mathrm{p}}(X R F)} \approx 6.25 \frac{40 \mathrm{keV}}{E_{\mathrm{p}}(X R F)}\langle\Delta t\rangle_{\mathrm{GRB}}$.

The durations of single pulses in XRFs, for which $\theta \geq$ $3.4 / \gamma$, should be, on average, longer than in GRBs by a factor $\geq 6.25$. The median duration of the single pulses of GRBs is $\overline{\Delta t}(G R B) \sim 0.92 \mathrm{~s}$ full width at half-maximum (Lee et al. 2000; McBreen et al. 2003). The prediction for XRFs is, therefore, $\overline{\Delta t}(X R F) \geq 5.75 \mathrm{~s}$.

\subsection{The total duration of XRFs}

The duration of a multi-pulse GRB or XRF is the total duration of the CBs' emission-times by the CB-emitting engine, which is independent of the viewing angle, plus the duration of the last pulse. On average, there are 6 significant pulses in a single GRB (Quilligan et al. 2002) and the interval between pulses is, on average, roughly twice a pulse's duration. Thus, it is a fair approximation (on average) to neglect a pulse's duration relative to the total duration of a GRB or an XRF. In that approximation, their total durations should be approximately the same. This agrees with the observations (Heise et al. 2000; in 't Zand et al. 2002; Kippen et al. 2002).

In contrast with the above, the duration of individual pulses is, on average, $\sim 6.25$ times longer in XRFs than in GRBs, as discussed in the previous subsection. The $1+z$ redshift dependence of the total- and pulse durations is the same, and it is common to XRFs and GRBs. Hence, XRFs should have a much less pronounced temporal structure and a much smaller "variability" than GRBs.

\subsection{XRF fluences}

The total energy emitted by a $\mathrm{CB}$ in its rest system, $\mathcal{E}_{\mathrm{CB}}^{\prime} \sim$ $0.8 \times 10^{44} \mathrm{erg}$ on average, is found to be very narrowly peaked 
around its central value ${ }^{3}$ : its dispersion is a factor $\sim 2$ either way (Dado et al. 2002a; Dar \& De Rújula 2003). Let $D_{L}(z)$ be the luminosity distance $(7.12 \mathrm{Gpc}$ at $z=1$, for the current cosmology with $\Omega=1, \Omega_{\Lambda}=0.7$ and $\left.H_{0}=65 \mathrm{~km} \mathrm{~s}^{-1} \mathrm{Mpc}^{-1}\right)$. Let $G(\theta) \approx 2\left(1+\theta^{4} \gamma^{4}\right) /\left(1+\theta^{2} \gamma^{2}\right)^{2}$ be the angular dependence of the low-energy Klein-Nishina cross section for Compton scattering, corresponding - in the large $\gamma$, small $\theta$ limit - to $\mathrm{d} \sigma / \mathrm{d} \cos \theta^{\prime} \propto 1+\cos ^{2} \theta^{\prime}$ in a CB's rest system.

In the fairly good standard-candle approximation, the observed energy fluence of a single CB is given by:

$F_{\text {Св }}=\frac{(1+z) G(\theta) \delta^{3}}{4 \pi D_{L}^{2}} \mathcal{E}_{\mathrm{CB}}^{\prime}$.

To a good approximation, $G=2$ for $\theta \gamma \geq 3$.4. Because of the strong $\delta$-dependence, the energy fluence of XRFs is a factor $\geq 6.25^{3} / 2 \approx 122$ smaller than that of GRBs, for which $G(\theta) \sim 1$.

In a sample of 35 XRFs and GRBs well localized with HETE II (Barraud et al. 2003), the mean energy fluence in the 7-400 keV band of 19 GRBs whose peak energy was well constrained by the observations, was $264 \times 10^{-7} \mathrm{erg} \mathrm{cm}^{-2}$. This is to be compared with $\sim 3 \times 10^{-7} \mathrm{erg} \mathrm{cm}^{-2}$ for the $7 \mathrm{XRFs}$ in the sample. The corresponding GRB-to-XRF fluence ratio is $\sim 83$, in satisfactory agreement with the expectation, particularly in view of the fact that the truncation of the contribution from $E_{\gamma} \geq 400 \mathrm{keV}$ subtracts a larger fraction from the GRBs' fluence than from the XRFs' fluence.

\subsection{Correlations}

The $\delta$-dependence is also strong in observables other than the fluence. To give a few examples, $\Delta t \propto 1 / \delta ; E_{\mathrm{p}} \propto \delta$; the photon-number fluence, $f$, is $\propto \delta^{2}$; the peak photon flux, $f_{\mathrm{p}}$, and the "isotropic" energy of a pulse, $\mathcal{E}^{\text {iso }}$, are $\propto \delta^{3}$; and the peak luminosity $L_{\mathrm{p}}$ (energy fluence per unit time) is $\propto \delta^{4}$ (Dar $\&$ De Rújula 2000, 2003). The powers of $\delta$ involved in these proportionality factors imply, among others, the following correlations:

$$
\begin{aligned}
& E_{\mathrm{p}} \propto[f]^{1 / 2} ; \quad \Delta t \propto\left[f_{\mathrm{p}}\right]^{-1 / 2}, \\
& E_{\mathrm{p}} \propto\left[f_{\mathrm{p}}\right]^{1 / 3} ; \quad \Delta t \propto\left[f_{\mathrm{p}}\right]^{-1 / 3}, \\
& E_{\mathrm{p}} \propto F^{1 / 3} ; \quad \Delta t \propto[F]^{-1 / 3}, \\
& E_{\mathrm{p}} \propto\left[\mathcal{E}^{\mathrm{iso}}\right]^{1 / 3} ; \quad \Delta t \propto\left[\mathcal{E}^{\mathrm{iso}}\right]^{-1 / 3}, \\
& E_{\mathrm{p}} \propto\left[L_{\mathrm{p}}\right]^{1 / 4} ; \quad \Delta t \propto\left[L_{\mathrm{p}}\right]^{-1 / 4} .
\end{aligned}
$$

The correlations in Eqs. (7) to (11) apply to individual pulses and pulse averages over a GRB or an XRF. The relations not involving time measures $\Delta t$ (which behave differently for pulses and inter-pulse delays) also apply to global XRF or GRB properties. All these correlations, are well satisfied for ordinary GRBs in particular for pulses of GRBs with known redshift, after correction for the $z$-dependence (Dar \& De Rújula 2003). At present, the low photon counting-levels in XRFs prevent a reliable resolution of XRFs into individual pulses, and the ensuing tests of most of the above correlations for single pulses.

\footnotetext{
3 In the CB model GRBs (and, more so, their individual pulses) are much better standard candles than in the FB models (Frail et al. 2001; Berger et al. 2003; Bloom et al. 2003b).
}

\subsection{The shape of single pulses}

In the $\mathrm{CB}$ model, to a rather good approximation, single GRB and XRF pulses have shapes that can be approximated by the simple expression:

$\frac{\mathrm{d} N}{\mathrm{~d} t}=\exp \left[-\left(\frac{t_{1}}{t}\right)^{m}\right]\left\{1-\exp \left[-\left(\frac{t_{2}}{t}\right)^{n}\right]\right\}$

where $t$ is the time from the beginning of the pulse (not of the GRB or XRF), $t_{1}$ and $t_{2}$ are the characteristic rise and fall times, $\left(t_{i}=O(\Delta t)\right.$ in Eq. (5) for XRFs), and $n$ and $m$ are power indices whose median values are near 2 . This pulse shape describes well the observed shape of single GRB pulses (Dar \& De Rújula 2003). At present, low statistics prevents a reliable resolution of XRFs into individual pulses and a test of this prediction.

\subsection{Spectral shape and spectral evolution}

In the $\mathrm{CB}$ model, the $\gamma$ rays of a GRB and the X-rays of an XRF are generated by inverse Compton scattering of "ambient light". This light permeates the semi-transparent, previously wind-fed surroundings of the parent star and results from their illumination by the early SN luminosity. The ambient light, as befits a semi-transparent, externallyenergized medium, has a thin thermal-bremsstrahlung spectrum $\mathrm{d} N / \mathrm{d} E_{i} \propto \exp \left(-E / T_{i}\right) / E$, with $T_{i}=O(1) \mathrm{eV}$. The ambient light is Compton up-scattered by the CBs' electrons, some of which are simply comoving with it, while others have been accelerated and Compton-cooled to a power-law distribution of index $\tilde{p} \sim 3.2$, by a "shockless acceleration" process studied in Frederiksen et al. (2003) and discussed in Dar \& De Rújula (2003). The result of the convolution - with an inverse Compton scattering kernel - of the electron and ambient light distributions is, to a very good approximation, given by the simple expression:

$$
\begin{aligned}
\frac{\mathrm{d} N}{\mathrm{~d} E} & \propto\left(\frac{T}{E}\right)^{\alpha} \mathrm{e}^{-E / T}+b\left(1-\mathrm{e}^{-E / T}\right)\left(\frac{T}{E}\right)^{\beta} \\
\alpha & \sim 1 ; \quad \beta=\frac{\tilde{p}+1}{2} \sim 2.1 .
\end{aligned}
$$

Here $b$ is a parameter reflecting the different normalizations of the comoving and accelerated electrons and

$T \equiv \frac{4}{3} T_{i} \frac{\gamma \delta}{1+z}\left\langle 1+\cos \theta_{i}\right\rangle$,

where $\left\langle\cos \theta_{i}\right\rangle$ is the mean angle of the ambient light photons relative to the radial direction towards the progenitor, reflecting the fact that, for a semitransparent wind, the ambient light may not be perfectly isotropic.

The spectrum of Eq. (13) is independent of the CB's expansion rate, its baryon number, its geometry and its density profile. Moreover, its derivation rested only on observations of the properties of the surroundings of exploding stars, Coulomb scattering, and an input electron-distribution extracted from numerical studies also based only on "first principles". The spectrum predicted in the CB model bears a striking resemblance to the phenomenological Band spectrum traditionally 


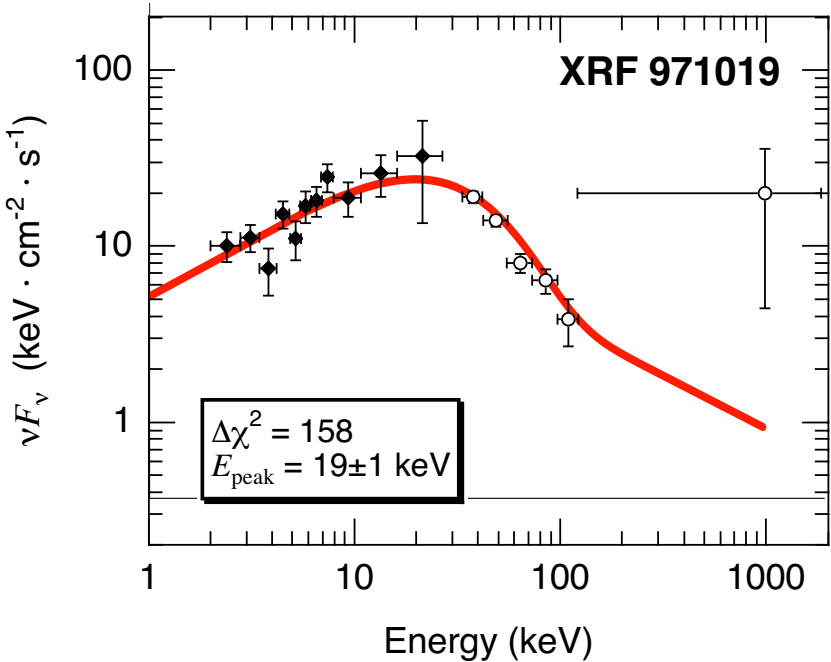

Fig. 1. The spectral data from BeppoSAX/WFC (solid diamonds) and CGRO/BATSE (open circles) on GRB 971019 (Kippen et al. 2002) and the CB-model fit of Eq. (13) to these data. The $\chi^{2}$ and $E_{\mathrm{p}}$ values are those of the Band fit. The CB-model fit has $T \sim E_{\mathrm{p}}$ and better $\chi^{2}$.

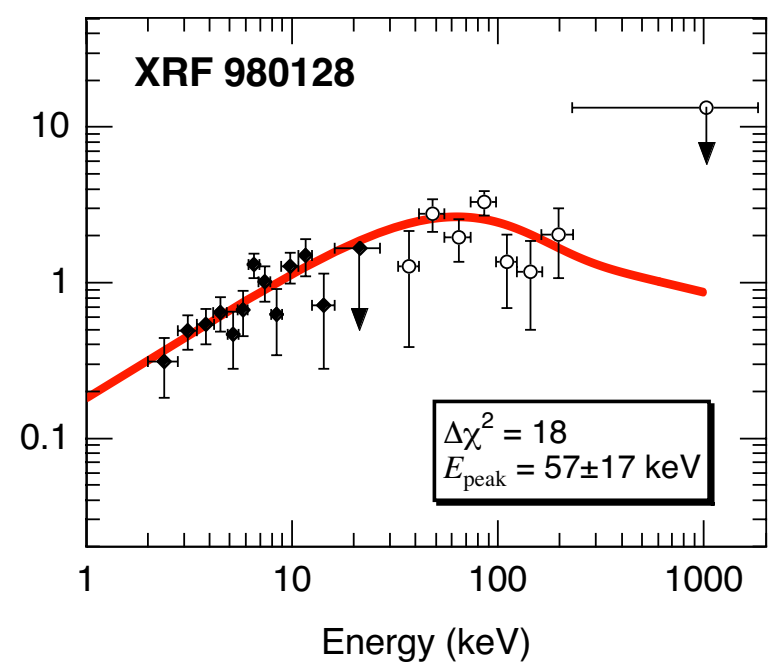

Fig. 2. The spectral data from BeppoSAX/WFC (solid diamonds) and CGRO/BATSE (open circles) on GRB 9810128 (Kippen et al. 2002) and the CB model fit of Eq. (13) to these data. The $\chi^{2}$ and $E_{\mathrm{p}}$ values are those of the Band fit. The CB-model fit has $T \sim E_{\mathrm{p}}$ and similar $\chi^{2}$.

used to describe GRB energy spectra (e.g., Band et al. 1993; Preece et al. 2000, for an analysis of BATSE data; Amati et al. 2002, for BeppoSAX data; and Barraud et al. 2003, for HETE II data). This spectrum also fits well the spectrum observed in XRFs as shown in Figs. 1-3. The parameters of the fits are, respectively, $T=20,50,30 \mathrm{keV}, b=0.2,0.7, \sim 0.01$, and $\beta=2.6,2.3,2.7$. The fits, in particular the last one, are quite insensitive to $b$.

\subsection{The time-energy correlations}

Such as we have treated it so far, the distribution $\mathrm{d} N / \mathrm{d} t \mathrm{~d} E$ of the X-rays in an XRF pulse - as a function of both time and energy - is a product of a function of only time, Eq. (12), and a function of only energy, Eq. (13). One reason for this is that we

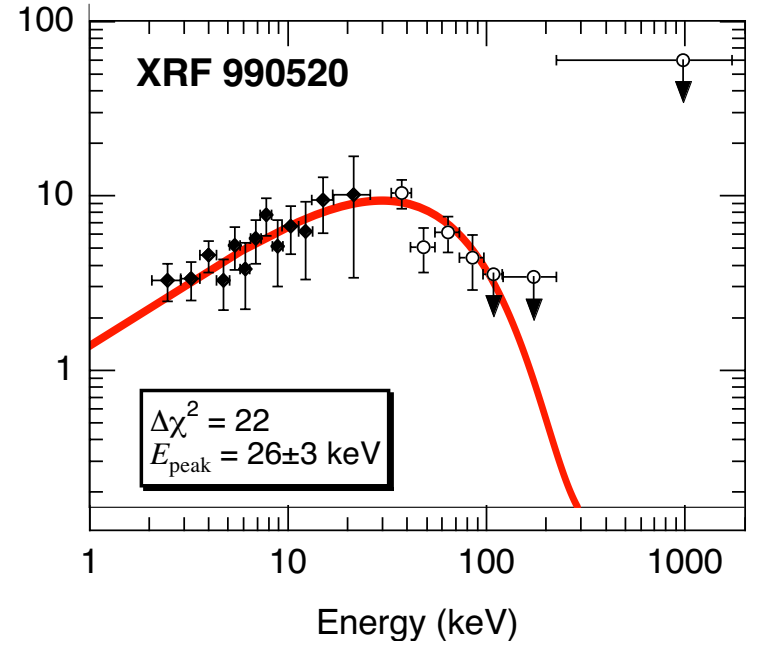

Fig. 3. The spectral data from BeppoSAX/WFC (solid diamonds) and CGRO/BATSE (open circles) on GRB 990520 (Kippen et al. 2002) and the $\mathrm{CB}$ model fit of Eq. (13) to these data. The $\chi^{2}$ and $E_{\mathrm{p}}$ values are those of the Band fit. The CB-model fit has $T \sim E_{\mathrm{p}}$ and similar $\chi^{2}$.

have not yet taken into account the fact that the cooling time of the accelerated electrons in a CB - by Compton scattering is of the same order of magnitude as the (Compton-scattering) transparency time of the $\mathrm{CB}$, which is the time characterizing a pulse's width (Dar \& De Rújula 2003). Consequently, the in$\operatorname{dex} \tilde{p}$ of the power-law electron energy distribution ought to evolve during a pulse from $\tilde{p} \sim 2.2$ to $\tilde{p} \sim 3.2$. Equivalently, the index $\beta$ in Eq. (13) is expected to vary from $\beta=\beta_{1} \sim 1.6$ to $\beta=\beta_{2} \sim 2.1$.

There is another fact contributing to a non-trivial correlation between energy and time within a GRB pulse. The relation between the "temperature" $T_{i}$ characterizing the initial ambient-light thermal-bremsstrahlung distribution and that of the resulting GRB or XRF photons, $T$, is that of Eq. (14). As the $\mathrm{CB}$ reaches the more transparent outskirts of the wind, its ambient light distribution is bound to become increasingly radial, meaning that the average $1+\cos \theta_{i}$ in Eq. (14) will depart from $\sim 1$ and tend to 0 as $1 / r^{2}$ : the point-source long-distance limit. Since this transition depends on the integrated absorption by a wind with $\rho \propto 1 / r^{2} \propto 1 / t^{2}$, it can be characterized by a simple time-dependence of the effective temperature in Eq. (13):

$T \rightarrow T(t) \sim T\left\{1-\exp \left[-\left(t_{T} / t\right)^{2}\right]\right\}$,

with $t_{T}$ of the same order as a pulse's width, or is somewhat smaller (Dar \& De Rújula 2003).

The two time-energy correlations we just discussed make pulses decrease in duration and peak earlier in time as the energy interval in which they are measured increases. They also imply that a pulse's spectrum gets softer as time elapses during a pulse. This behaviour is observed in GRBs (e.g., Fenimore et al. 1995; Norris et al. 1996; Ramirez-Ruiz \& Fenimore 2000; Wu \& Fenimore 2000; Golenetskii et al. 1983; Bhat et al. 1994). We expect XRFs to display precisely the same trends. The spectral evolution in a GRB and XRF pulse is mainly due to the decline of the effective temperature described by 
Eq. (15). This implies that the width of a GRB or an XRF pulse, dominated by its late-time behaviour, changes as it is measured in different energy bands approximately as:

$\Delta t \propto E^{-\kappa}, \quad \kappa \leq 0.5$,

where $\kappa=0.5$ is the limiting value for an exact $T \propto 1 / t^{2}$. This result is in agreement with the observationally inferred relation $t_{\text {FWHм }} \propto E^{-0.43 \pm 0.10}$ for the average full width at half-maximum of GRB pulses as a function of the energies of the four BATSE channels (Fenimore et al. 1995; Norris et al. 1996). We expect this result to be valid for XRF pulses as well.

\section{The afterglow of XRFs}

In the CB model, the AGs of GRBs and XRFs consist of three contributions, from the CBs themselves, the concomitant $\mathrm{SN}$, and the host galaxy:

$F_{\mathrm{AG}}=F_{\mathrm{CBs}}+F_{\mathrm{SN}}+F_{\mathrm{HG}}$,

the latter contribution being usually determined by late-time observations, when the CB and SN contributions become negligible.

Let the unattenuated energy flux density of SN1998bw at redshift $z_{\text {bw }}=0.0085$ (Galama et al. 1998) be $F_{\text {bw }}[v, t]$. For a similar SN placed at a redshift $z$ (Dar 1999; Dar \& De Rújula 2000):

$F_{\mathrm{SN}}[v, t]=\frac{1+z}{1+z_{\mathrm{bw}}} \frac{D_{\mathrm{L}}^{2}\left(z_{\mathrm{bw}}\right)}{D_{\mathrm{L}}^{2}(z)} A(v, z) F_{\mathrm{bw}}\left[v^{\prime}, t^{\prime}\right]$,

where $D_{\mathrm{L}}(z)$ is the luminosity distance, $A(v, z)$ is the attenuation along the line of sight, $v^{\prime}=v(1+z) /\left(1+z_{\mathrm{bw}}\right)$, and $t^{\prime}=t(1+$ $\left.z_{\text {bw }}\right) /(1+z)$.

The evolution of $F_{\mathrm{CBs}}$ with time is due to the deceleration of the CBs in the ISM. In an approximately hydrogenic ISM of constant number density $n_{\mathrm{p}}$, the function $\gamma(t)$ is determined by energy-momentum conservation to be the real root of the cubic:

$\frac{1}{\gamma^{3}}-\frac{1}{\gamma_{0}^{3}}+3 \theta^{2}\left[\frac{1}{\gamma}-\frac{1}{\gamma_{0}}\right]=\frac{6 c t}{(1+z) x_{\infty}}$

where $\gamma_{0}$ - previously called simply $\gamma-$ is the initial Lorentz factor of a CB (very close to being constant during the GRB or $\mathrm{XRF}$ emission), $t$ is the observer's time and

$x_{\infty} \equiv \frac{N_{\mathrm{B}}}{\pi R_{\infty}^{2} n_{\mathrm{p}}}$,

with $N_{\mathrm{B}}$ being the baryon number of the CB and $R_{\infty}$ its calculable asymptotic radius, reached within minutes of observer's time (Dado et al. 2002a). It takes a distance $x_{\infty} / \gamma_{0}$, typically a fraction of a kpc, for the $\mathrm{CB}$ to half its original Lorentz factor.

The AG of the CBs is mainly due to synchrotron radiation from accelerated electrons in the CB's chaotic magnetic field. It has the approximate form (Dado et al. 2003e):

$F_{\mathrm{CB}}[v, t] \propto n^{(1+\hat{\alpha}) / 2} R_{\infty}^{2} \gamma^{3 \hat{\alpha}-1} \delta^{3+\hat{\alpha}} A(v, t) v^{-\hat{\alpha}}$, with $\hat{\alpha}$ changing from $\sim 0.5$ to $\sim 1.1$ as the emitted frequency ${ }^{4}$ in the CB's rest frame crosses the "injection bend",

$v_{\mathrm{b}}(t) \simeq 1.87 \times 10^{3}[\gamma(t)]^{3}\left[\frac{n_{\mathrm{p}}}{10^{-3} \mathrm{~cm}^{3}}\right]^{1 / 2} \mathrm{~Hz}$.

By the time $\gamma(t)=\gamma_{0} / 2, F_{\mathrm{CB}}[v, t]$ is orders of magnitude smaller than $F_{\mathrm{CB}}[v, 0]$.

The attenuation $A(v, t)$ is a product of the attenuation in the host galaxy, in the intergalactic medium, and in our Galaxy. The attenuation in our galaxy in the direction of the GRB or XRF is usually estimated from the Galactic maps of selective extinction, $E(B-V)$, of Schlegel et al. (1998), using the extinction functions of Cardelli et al. (1986). The extinction in the host galaxy and the intergalactic medium, $A(v, t)$ in Eq. (18), can be estimated from the difference between the observed spectral index at very early time when the CBs are still near the $S N$ and that expected in the absence of extinction. Indeed, the CB model predicts - and the data confirm with precision - the gradual evolution of the effective optical spectral index towards the constant value $\approx-1.1$ observed in all "late" AGs (Dado et al. 2002a, 2003a). The "late" index is independent of the attenuation in the host galaxy, since at $t>1$ (observer's) days after the explosion, the CBs are typically already moving in the low-column-density, optically-transparent halo of the host galaxy.

The comparison of the predictions of Eq. (21) with the observations of optical and X-ray AG light curves and of broadband spectra is discussed in Dado et al. (2002a, 2003a), respectively. The results - for all GRBs of known redshift - are very satisfactory and involve a total of only five parameters (two of which, $z$ and $\theta$ are not "intrinsic" to the model).

The initial value of $\delta$ of XRFs is, on the average, smaller by a factor $\geq 6.25$ than its mean value in GRBs. According to Eq. (21), this implies that the early-time optical AG of XRFs should be flatter than that of long-duration GRBs, dimmer by a factor of a few hundred, and stretched in time by a factor of a few. At late time, when $[\gamma(t) \theta]^{2} \ll 1$, Eq. (1) implies that $\delta(t) \approx 2 \gamma(t)$, while Eq. (19) implies that when $3[\gamma(t) \theta]^{2} \ll 1, \gamma(t)$ approaches its asymptotic behaviour, $\gamma(t) \approx\left[2 c t / 3(1+z) x_{\infty}\right]^{-1 / 3}$. Consequently, the late time AGs of GRBs and XRFs are similar and have the asymptotic behaviour $F_{v} \sim v^{-1.1 \pm 0.1} t^{-2.13 \pm 0.1}$ (Dado et al. 2002a). Because of the initially small Doppler factor, the optical AGs of XRFs may already be above the injection bend at early time, yielding $\mathrm{a} \sim v^{-1.1}$ optical spectrum (before extinction).

The thresholds of GRB and XRF detectors and the strong decline of the peak luminosity with viewing angle imply that most observed XRFs should have relatively low redshifts. This may partly compensate for the initial low flux of their AGs and, combined with their relatively slow decline, make it easier to follow their late-time AGs, particularly at radio frequencies. Because of their relative proximity, we expect the AGs of most XRFs to include a detectable SN1998bw-like contribution, if not overshined by the CBs' AG and/or by the host galaxy, and

\footnotetext{
${ }^{4}$ In the CB model, the spectral evolution as $\hat{\alpha}$ changes from $\sim 0.5$ to $\sim 1.1$ is interpolated by $\left(v / v_{\mathrm{b}}(t)\right)^{-0.5} / \sqrt{1+\left[v / v_{\mathrm{b}}(t)\right]^{1.1}}$ (Dado et al. 2003a).
} 
if not strongly extinct by dust in the host or our galaxy. Such a SN contribution would add evidence to our claim that XRFs and GRBs are one and the same. A signature even more specific to the CB model would be an observed superluminal motion of their CBs (Dar \& De Rújula 2000a).

So far, only the light curves of the AGs of XRFs 020903 and 030723 have been measured and reported in sufficient detail to allow comparisons with the CB model. We describe them next.

\section{XRF 020903}

This XRF was detected and localized with HETE II (Ricker et al. 2002). Its AG was discovered and followed by Soderberg et al. (2002b). Subsequent photometric observations were reported by Covino et al. (2002) and Gorosabel et al. (2002). Spectroscopic observations by Hamuy and Shectman obtained with the Magellan $6.5 \mathrm{~m}$ telescope 25 days after burst revealed narrow emission lines from an underlying host galaxy at a mean redshift, $z=0.25$ (Soderberg 2002). Subtraction of the emission lines revealed a continuum that is consistent with a SN akin to $1998 \mathrm{bw}$ at $z=0.25$ (Soderberg: http: //www . astro. caltech. edu/ams/XrF.html).

The lightcurve of XRF 020903 showed a double peak structure (Sakamoto et al. 2003, to be published), which implies, in the CB model, that it was dominated by two CBs. Due to sparse observational data, a CB model fit with two independent $\mathrm{CBs}$ does not constrain enough the fitted parameters. Therefore, we have fitted the observations assuming a single $\mathrm{CB}$, which should be a good approximation if the two $\mathrm{CBs}$ had similar properties. Our CB-model fit to the broadband optical AG of this XRF is shown in Fig. 4 and the separate contributions of the $\mathrm{CB}$, the host galaxy and a 1998bw-like SN at $z=0.25$ are shown in Fig. 5. Extinction in our Galaxy in the direction of XRF 020903 was included, using $E(B-V)=0.032$ Schlegel et al. (1998), and the phenomenological relations of Cardelli et al. (1998), which result in the attenuation coefficients, $A(I)=0.89, A(R)=0.83$, $A(V)=0.78$, and $A(B)=0.72$. Because of lack of spectral information on the early $\mathrm{AG}$, the extinction of the $\mathrm{SN}$ in the host galaxy was taken to be equal to the typical extinction in our Galaxy, i.e. $E(B-V)=0.06$ with the extinction relations of Cardelli et al. (1998). The host galaxy was assumed to have the spectrum of a template star burst galaxy, as measured e.g. by Gorosabel et al. (2003) for GRB 000210, with a spectral break around $4000(1+z) \AA$, and $F_{v}$ approximately constant at longer wavelengths. The fitted values of the $\mathrm{CB}$ model parameters are, $\gamma=1693, \theta=3.66 \mathrm{mrad}, n_{\mathrm{p}}=3.5 \times 10^{-2} \mathrm{~cm}^{-3}$ and $x_{\infty}=87 \mathrm{kpc}$. These parameters are all in the corresponding ranges characteristic of GRBs, but for $\theta$ which, as expected, is larger than for all of the 32 GRBs we have analyzed, but GRB 980425.

The fact that GRB 980425 - which, as we discussed, is marginally an XRF - had an even larger observer's angle than XRF 020903 is what explains the greater luminosity of the radio AG of the XRF. The direct comparison of optical AGs is not an issue because that of XRF 020903 is due to the CBs, while the very large observation angle of GRB 980425 implied

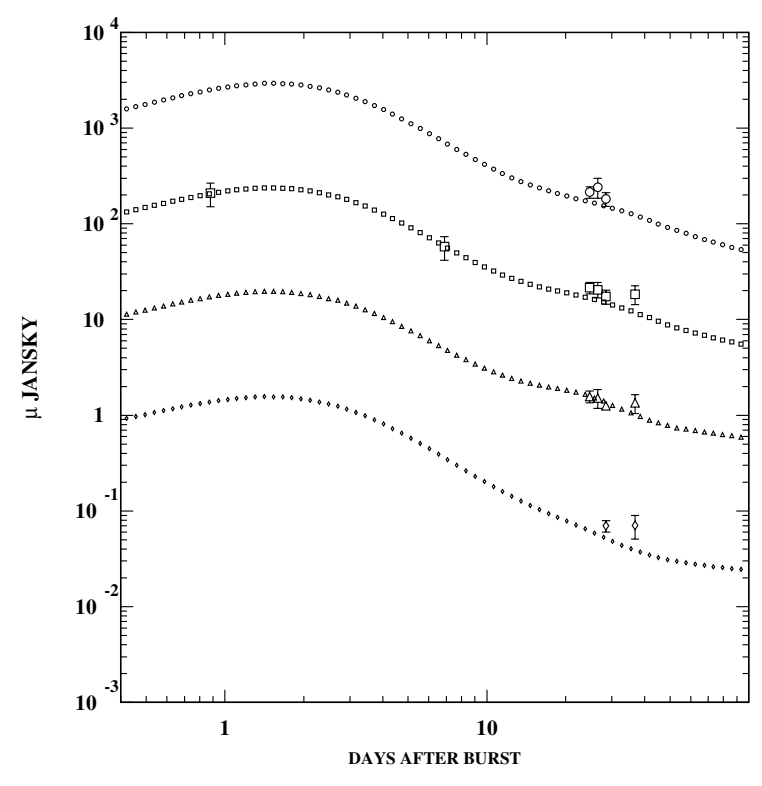

Fig. 4. CB-model fit to the measured (top to bottom) $I, R, V$, and $B$-band AGs of XRF 020903, multiplied by 10, 1, 1/10, 1/100, respectively. The theoretical contribution from a SN1998bw-like supernova at $z=0.25$ was corrected for the known extinction in the Galaxy in the direction of XRF 020903. Extinction in the host galaxy was neglected due to lack of information on the early time AG. A contribution of the host galaxy was included assuming a spectrum of a typical star-burst galaxy. The SN contribution is not directly visible in the light curves.

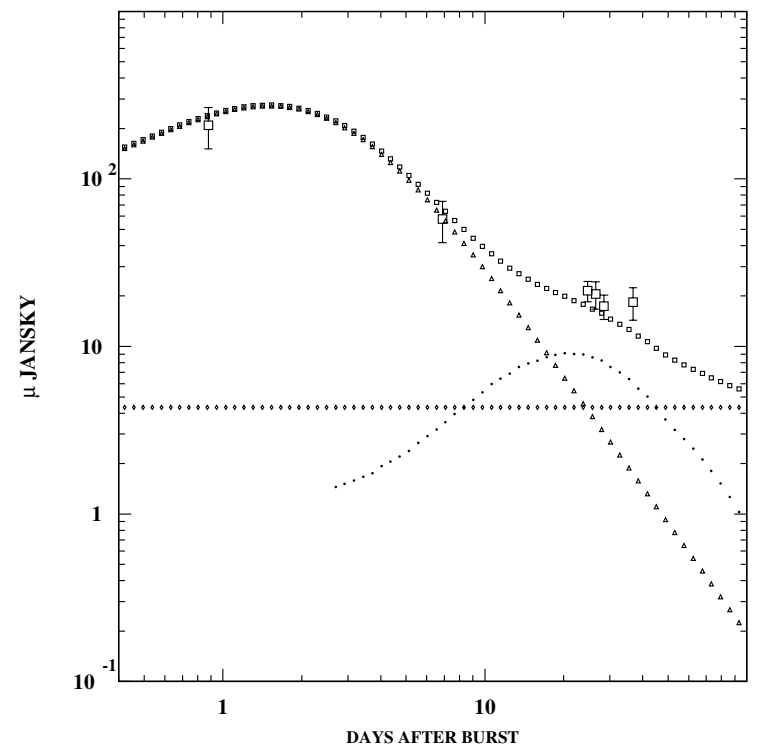

Fig. 5. The $R$-band AG of XRF 020903. Triangles: $F_{\mathrm{CBs}}$, from the fit to the broad-band AG. Dots: $F_{\mathrm{SN}}$, a $1998 \mathrm{bw}-$ like $\mathrm{SN}$ at $z=0.25$, corrected for Galactic extinction in the direction of XRF 020903 but not for extinction in the host galaxy. Diamonds: the host galaxy, assumed to have the typical spectrum of a star-burst galaxy. Squares: total AG. The SN contribution is not clearly visible in the light curve though it dominates, in the $\mathrm{CB}$ model, the late time AG.

that its optical AG was dominated by the SN (Dar \& De Rujula 2000).

The poorly measured light curve of the AG of XRF 020903 does not provide a convincing evidence for an underlying SN in XRF 020903, which dominates its late time behaviour in the 


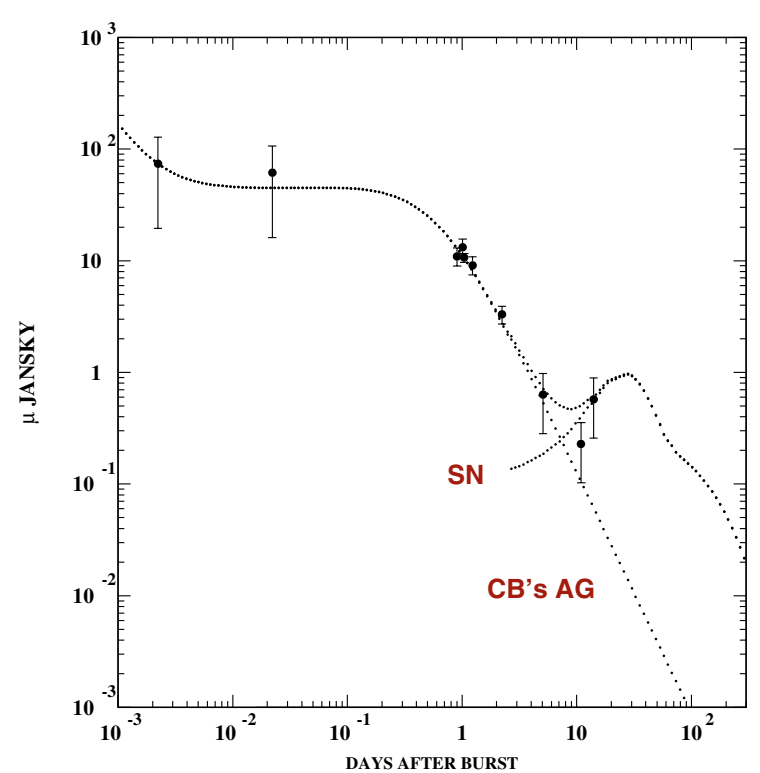

Fig. 6. The CB-model fit to the $R$-band AG of XRF 030723 (Prigozhin et al. 2003; Fox et al. 2003b; Dullighan et al. 2003a,b; Smith et al. 2003; Bond et al. 2003; Fynbo et al. 2003). The first two data points have been deduced from the unfiltered measurements of Smith et al. (2003), assuming an early $F_{v} \sim v^{-0.5}$ (Dado et al. 2003a). All errors were multiplied by a factor 2 to account for cross-calibration uncertainties. The observed "rebrightening" 14 days after the XRF may have been due to the contribution of a SN (Fynbo et al. 2003). In the CB-model's prediction, the redshift of the 1998bw-like SN has been adjusted to the normalization of the late-time points; without extinction corrections in the host galaxy or ours, it corresponds to a redshift of $z \sim 0.75$.

CB model, as can be seen from Fig. 5. However, precise new measurements of the spectrum of the host galaxy are highly desirable. When subtracted from the spectrum of the AG measured by Hamuy and Shectman on day 25 after the burst, they may provide the decisive spectroscopic evidence for an underlying SN akin to 1998bw in the AG of XRF 020903 (Soderberg et al. 2002).

The equivalent isotropic $\gamma$-ray energy of XRF 020903 was estimated to be $E_{\text {iso }}^{\gamma} \sim 1-2 \times 10^{50}$ erg (Soderberg: http://www.astro.caltech.edu/ams/XrF.html). The quoted CB-model fitted parameters result in $\delta(t=0) \approx 87$. The light curve of XRF 020903 appears to be dominated by two CBs (Sakamoto et al. 2003, to be published). Thus, the CB-model prediction for its equivalent $\gamma$-ray energy is, $E_{\text {iso }}^{\gamma}=2 \delta^{3} \mathcal{E}_{\mathrm{CB}}^{\prime} \approx 2.1 \times 10^{50} \mathrm{erg}$, in good agreement with its observationally-inferred value.

\section{XRF 030723}

This XRF was detected and localized with HETE II (Prigozhin et al. 2003). Its AG was discovered by Fox et al. (2003b). Later measurements were reported by Dullighan et al. (2003a,b), Smith et al. (2003), Bond et al. (2003) and Fynbo et al. (2003) who reported a "rebrightening" in the optical AG 14 days after the XRF, which may be due to the contribution of a SN. This rebrightening can be seen in Fig. 6, in which we also present the $\mathrm{CB}$ model fit to the AG. The normalization of the 1998bw-like SN contribution has been adjusted: without extinction corrections in the host galaxy or ours, it corresponds to a redshift of $z \sim 0.75$ (the redshift of this XRF has not been measured so far). The fitted values of the CB-model parameters are $\gamma=776, \theta=2.79 \mathrm{mrad}, n_{\mathrm{p}}=0.121 \mathrm{~cm}^{-3}$ and $x_{\infty}=29.5 \mathrm{kpc}$. Once again, only $\theta$ is outside the characteristic range of GRB parameters in the $\mathrm{CB}$ model. Late-time measurements of the spectrum of the host galaxy and its redshift, as well as colour photometry and precise spectroscopy of the late-time AG are needed to verify whether or not the rebrightening is due to a SN akin to SN1998bw at the XRF position.

\section{Conclusions}

In Dar \& De Rújula (2000) we argued that long-duration GRBs may all be associated with core-collapse SNe akin to SN1998bw when viewed near axis, and that GRB 980425 (nearly an XRF) was a normal GRB viewed at a much larger angle than ordinary GRBs. In a series of papers (Dado et al. 2002a,b,c, 2003a,b,c) we presented supporting evidence for the GRB/SN association from a CB-model analysis of the AGs of all GRBs of known redshift. The recent spectroscopic evidence for a GRB/SN association in the AGs of GRBs 030329 (Stanek et al. 2003; Hjorth et al. 2003; Matheson et al. 2003) and GRB 021211 (Della Valle et al. 2003) is perhaps the most convincing evidence.

In Dar \& De Rújula (2003) we argued that short-duration GRBs may be associated with SNe of type Ia, and that XRFs are ordinary GRBs, which - like GRB 980425 - are viewed from much larger angles. In this paper we have demonstrated that all the currently-known properties of XRFs, including the $\mathrm{XRF} / \mathrm{SN}$ association, support this interpretation. If our contentions are correct, it follows that short XRFs - unobserved to date - should have properties that scale relative to those of short GRBs with the same scaling laws which relate XRFs to long GRBs. The association of short XRFs and short GRBs with SNe - allegedly of type Ia and akin to SN1997cy - is, so far, purely conjectural (Dar \& De Rújula 2003).

At the moment, the best proof of our alleged XRF/SN association may be provided by obtaining accurate spectra of the host galaxies of XRFs 020903 and 030723 , now that the putative SN and the AG have faded away. These spectra may be subtracted, respectively, from the spectrum of XRF 020903 taken with the Magellan 6.5 m telescope on September 28.1 UT 2002 (Hamuy \& Shectman 2002, to be published) near the expected SN maximum, and from the spectrum of the AG of XRF 030723, which was measured with the VLT around the SN maximum (Fynbo et al. to be published). After subtraction of the galaxies' contributions, the spectra may expose the underlying SNe.

Note added in proff: After this article was posted in the astroph archives (Dado et al., arXiv: astro-ph/0309294), Lamb et al. reported there [arXiv:astro-ph/0309456] the peak energy, $E_{\mathrm{p}}$, of 14 XRFs localized by HETE II. Out of these 14 cases, 6 have $E_{\mathrm{p}}$ values smaller than those observed by BeppoSAX and BATSE by about an order of magnitude. Their fluence and isotropic equivalent energies are much larger than 
those implied by Eqs. (9) and (10). We contend that the correct $E_{\mathrm{p}}$ values for these XRFs are larger, and are similar to those observed by BeppoSax (Heise et al. 2001) and BATSE (Kippen et al. 2002). The very low photon fluences detected in these XRFs by HETE II above $25 \mathrm{keV}$ - as well as the unknown column density of absorbing material along the line of sight - must have prevented a correct determination of the true values of $E_{\mathrm{p}}$. Moreover, Lamb et al. (2003) did not include GRB 980425 in their plot (Fig. 1.2) of $E_{\mathrm{p}}$ as a function of $\mathcal{E}^{\text {iso }}$. The result for this GRB badly violates their advocated relation, $E_{\mathrm{p}} \propto\left[\mathcal{E}^{\mathrm{iso}}\right]^{1 / 2}$, but is consistent with the $\mathrm{CB}$ model relation: $E_{\mathrm{p}} \propto\left[\mathcal{E}^{\mathrm{iso}}\right]^{1 / 3}$.

Acknowledgements. This research was supported in part by the Helen Asher Space Research Fund at the Technion. Observational informations communicated to us by J. Fynbo, M. Hamuy and A. Soderberg prior to their publication are gratefully acknowledged. One of us, Arnon Dar, is grateful for the hospitality extended to him at the CERN Theory Division.

\section{References}

Amati, L., Frontera, F., Tavani, M., et al. 2002, A\&A, 390, 81 Band, D., Matteson, J., Ford, L., et al. 1993, ApJ, 413, 281 Barraud, C., Olive, J. F., Lestrade, J. P., et al. 2003, A\&A, 400, 1021 Berger, E., Kulkarni, S., \& Frail, D. A. 2003, ApJ, 590, 379 Bhat, P. N., Fishman, G. J., Meegan, C. A., et al. 1994, ApJ, 426, 604 Bloom, J. S., Frail, D. A., \& Kulkarni, S. R. 2003, ApJ, 594, 674, Bloom, J. S., Fox, D. W., van Dokkum, P. G., et al. 2003b, ApJ, 599, 957

Bond, H. E., Henden, A., Levay, Z. G., et al. 2003, Nature, 422, 425 Cardelli, J. A., Clayton, G. C., \& Mathis, J. S. 1988, ApJ, 329, L33 Covino, S., Malesani, D., Ghisellini, G., et al. 2002, GCN Circ. 1563 Dado, S., Dar, A., \& De Rújula, A. 2002a, A\&A, 388, 1079 Dado, S., Dar, A., \& De Rújula, A. 2002b, ApJ, 572, L143 Dado, S., Dar, A., \& De Rújula, A. 2002c, A\&A, 393, L25 Dado, S., Dar, A., \& De Rújula, A. 2003a, A\&A, 401, 243 Dado, S., Dar, A., \& De Rújula, A. 2003b, ApJ, 593, 961 Dado, S., Dar, A., \& De Rújula, A. 2003c, ApJ, 594, L89 Dado, S., Dar A., \& De Rújula A. 2003d, Phys. Lett. B, 562, 161 Dar, A. 1998, ApJ, 500, L93

Dar, A. 1999, A\&AS, 138(3), 505

Dar, A. 2003 [arXiv: astro-ph/0301389]

Dar, A., \& De Rújula, A. 2000 [arXiv: astro-ph/0008474]

Dar, A., \& De Rújula, A. 2003 [arXiv:astro-ph/0308248]

Dar, A., \& Plaga, R. 1999, A\&A, 349, 259

Della Valle, M., Malesani, D., Benetti, S., et al. 2003, A\&A, 406, L33

De Rújula, A. 1987, Phys. Lett., 193, 514

De Rújula, A. 2002 [arXiv: astro-ph/0207033]

De Rújula, A. 2003 [hep-ph/0306140]

Dullighan, A., Butler, N., Vanderspek, R., Villasenor, J., \& Ricker, G. 2003a, GCN Circ. 2326

Dullighan, A., Butler, N., Vanderspek, R., Villasenor, J., \& Ricker, G. 2003b, GCN Circ. 2336

Fenimore, E. E., in 't Zand, J., Norris, J. P., Bonnell, J. T., \& Nemiroff, R. J. 1995, ApJ, 448, L101

Frail, D. A., Kulkarni, S. R., Sari, R, et al. 2001, ApJ, 562, L55

Frederiksen, J. T., Hededal, C. B., Haugbolle, T., \& Nordlund, A. 2003 [arXiv: astro-ph/0303360]

Fruchter, A., Pattel, S., Kouveliotou, C., et al. 2002, GCN Circ. 1268

Fynbo, P. U., Hjorth, J., \& Gorosabel, J. 2003, GCN Circ. 2345
Galama, T. J., Vreeswijk, P. M., van Paradijs, J., et al. 1998, Nature, 395,670

Gandolfi, G. 2001, GCN Circ. 1118

Garnavich, P. M., et al. 2003b, IAU Circ. 8108

Golenetskii, S. V., Mazets, E. P., Aptekar, R. L., \& Ilinskii, V. N. 1983, Nature, 306, 451

Gorosabel, J., Hjorth, J., Pedersen, H., et al. 2002, GCN Circ. 1631

Gorosabel, J., Christensen, L., Hjorth, J., et al. 2003, A\&A, 400, 127

Granot, J., Panaitescu, A., Kumar, P., \& Woosley, S. E. 2002, ApJ, 570, L61

Hjorth, J., Sollerman, J., Moller, P., et al. 2003, Nature, 423, 847

Harrison, F. A., Yost, S., Fox, D. W., et al. 2001, GCN Circ. 1143

Heise, J., in't Zand, J., Kippen, R. M., \& Woods, P. M. 2001, Proc. 2nd Rome Workshop: GRBs in the Afterglow Era (Oct. 2000), ed. E. Costa, F. Frontera, \& J. Hjorth (Berlin: Springer)

Iwamoto, K., Mazzali, P. A., Nomoto, K., et al. 1998, Nature, 395, 672 Jin, Z. P., \& Wei, D. M. 2003 [arXiv: astro-ph/0308061]

Kippen, R. M., Woods, P. M., Heise, J., et al. 2003, AIP Conf. Proc., 662,244

Lazzati, D., Rossi, E., Ghisellini, G., \& Rees, M. J. 2004, MNRAS, 347, L1

Lee, A., Bloom, E. D., \& Petrosian, V. 2000, ApJS, 131, 1

McBreen, S., Quilligan, F., McBreen, B., Hanlon, L., \& Watson, D. 2003, AIP Conf. Proc., 662, 280

Matheson, T., Garnavich, P. M., \& Stanek, K. Z. 2003, ApJ, 599, 394

Norris, J. P., Nemiroff, R. J., Bonnell, J. T., et al. 1996, ApJ, 459, 393

Mirabel, I. F., \& Rodriguez, L. F. 1999, ARA\&A, 37, 409

Panaitescu, A., \& Kumar, P. 2001, ApJ, 560L, 49

Panaitescu, A., \& Kumar, P. 2003, ApJ, 592, 390

Piran, T. 2000, Phys. Rep., 333, 529

Plaga, R. 2001, A\&A, 370, 351

Preece, R. D., Briggs, M. S., Mallozzi, R. S., et al. 2000, ApJS, 126, 19

Prigozhin, G., Butler, N., Crew, G., et al. 2003, GCN Circ. 2313

Quilligan, F., McBreen, B., Hanlon, L., et al. 2002, A\&A, 385, 377

Ramirez-Ruiz, E., \& Fenimore, E. E. 2000, ApJ, 539, 12

Rhoads, J. E. 1997, ApJ, 487, L1

Rhoads, J. E. 1999, ApJ, 525, 737

Ricker, G., Atteia, J. L., Kawai, N., et al. 2002, GCN Circ. 1530

Rodriguez, L. F., \& Mirabel, I. F. 1999, ApJ, 511, 398

Rossi, E., Lazzati, D., \& Rees, M. J. 2002, MNRAS, 332, 945

Salmonson, J. D. 2003, ApJ, 592, 1002

Sari, R., Piran, T., \& Narayan, R. 1998, ApJ, 497, L17

Sari, R., Piran, T., \& Halpern, J. P. 1999, 519, L17

Schlegel, D. J., Finkbeiner, D. P., \& Davis, M. 1998, ApJ, 500, 525

Shaviv, N. J., \& Dar, A. 1995, ApJ, 447, 863

Smith, D. A., Akerlof, C. W., Quimby, R., et al. 2003, GCN Circ. 2338

Soderberg, A. M., Price, P. A., Fox, D. W., et al. 2002, GCN Circ. 1554

Stanek, K. Z., Matheson, T., Garnavich, P. M., et al. 2003, ApJ, 591, L17

Taylor, G. B., Frail, D. A., Kulkarni, S. R., et al. 2001, GCN Circ. 1136

Waxman, E. 2003b, Nature, 423, 388

Woosley, S. E., \& MacFadyen, A. I. 1999, A\&AS, 138, 499

Wu, B., \& Fenimore, E. E. 2000, ApJ, 535, L29

Yamazaki, R., Ioka, K., \& Nakamura, T. 2002, ApJ, 571, L31

Yamazaki, R. Yonetoku, D., \& Nakamura, T. 2003a, ApJ, 594, L79

Yamazaki, R., Ioka, K., \& Nakamura, T. 2003b, ApJ, 593, 941

in 't Zand, J., Reali, F., Granatain, S., et al. 2002, GCN Circ. 1383

Zhang, B., \& Mészáros, P. 2002, ApJ, 566, 712 